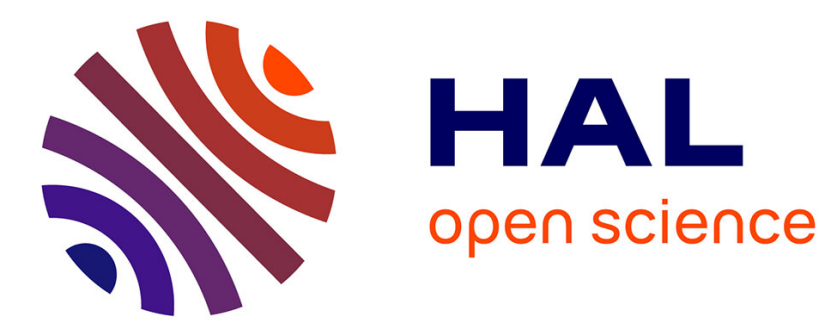

\title{
Sample environment in experiments using X-ray synchrotron radiation
}

\author{
B. Buras
}

\section{To cite this version:}

B. Buras. Sample environment in experiments using X-ray synchrotron radiation. Revue de Physique Appliquée, 1984, 19 (9), pp.697-703. 10.1051/rphysap:01984001909069700 . jpa-00245240

\section{HAL Id: jpa-00245240 https://hal.science/jpa-00245240}

Submitted on 1 Jan 1984

HAL is a multi-disciplinary open access archive for the deposit and dissemination of scientific research documents, whether they are published or not. The documents may come from teaching and research institutions in France or abroad, or from public or private research centers.
L'archive ouverte pluridisciplinaire HAL, est destinée au dépôt et à la diffusion de documents scientifiques de niveau recherche, publiés ou non, émanant des établissements d'enseignement et de recherche français ou étrangers, des laboratoires publics ou privés. 


\title{
Sample environment in experiments using $\mathbf{X}$-ray synchrotron radiation
}

\author{
B. Buras (*) \\ European Synchrotron Radiation Project, c/o CERN, CH-1211 Geneva 23, Switzerland
}

\begin{abstract}
Résumé. - Les anneaux de stockage à électrons (ou à positrons) modernes sont capables d'émettre des rayons $\mathrm{X}$ très intenses dans un spectre continu qui s'étend jusqu'à $0,1 \AA$ A. Cette lumière peut être produite dans les aimants de courbure ou dans des wigglers multipoles et wavelength shifters (écarteurs de longueur d'onde) installés dans les sections droites de l'anneau. Elle peut servir de façon immédiate aux expériences utilisant la lumière blanche et/ou après monochromatisation, aux expériences utilisant un faisceau de lumière monochromatique, dont la longueur d'onde peut être choisie à volonté dans le spectre continu émis. L'onduleur, un autre dispositif pour sections droites, produit du rayonnement quasi monochromatique. Ces dispositifs installés dans les sections droites (wigglers, wavelength shifters et onduleurs) permettent d'adapter le rayonnement synchrotron émis aux demandes de l'utilisateur et peuvent donc être considérés comme premier élément optique de la ligne. Cet aspect est particulièrement important pour les expériences avec échantillons dans un environnement spécial parce que ceux-ci imposent des restrictions et sur les expériences de diffusion et sur les expériences d'absorption. Néanmoins, ces limitations peuvent être minimisées pour chaque cas particulier en trouvant la meilleure combinaison d'environnement d'échantillons spéciaux, de méthode expérimentale employée, et du faisceau de rayonnement $\mathrm{X}$ adapté à l'expérience en ce qui concerne longueur d'onde, intensité, section efficace, divergence et polarisation. Ceci est discuté en quelque détail et illustré par des exemples.
\end{abstract}

\begin{abstract}
Modern electron (positron) storage rings are able to emit very intense X-ray radiation with a continuous spectrum extending to $0.1 \AA$, from bending magnets and insertion devices (wavelength shifters and multipole wigglers). It can be used directly for white beam experiments and/or for monochromatic beam experiments with wavelength chosen at will from the continuous spectrum. Another type of insertion device, called undulator produces quasi-monochromatic radiation. The insertion devices enable the tailoring of the emitted S.R. to the requirements of the users and can be treated as the first optical element of the beam line.

This feature is especially important for experiments with samples in special environment because the latter imposes limitations both on scattering and absorption experiments. However, these limitations can be minimized in each case by finding the best match between the design of the special environment, the experimental method used, and the X-ray beam tailored to the experiment with respect to wavelength. intensity, cross-section, divergence and polarization. This is discussed in some detail and illustrated by examples.
\end{abstract}

\section{Introduction.}

The special environment (e.g. a high pressure cell, a cryostat, a high temperature furnace) imposes in general limitations on the X-ray studies. These limitations can be minimised by finding the best possible match between the design of the special environment, the experimental method used, and the X-ray source. The main goal of this paper is to show that an X-ray synchrotron radiation source is a very flexible source which can be tailored individually to the requirements of the user and thus make it relatively easy to achieve

$\left(^{*}\right)$ On leave from Copenhagen University and Risoe National Laboratory, Denmark. a good match of the above mentioned requirements. The paper is arranged in the following way. Section 2 describes briefly the main features of synchrotron radiation (S.R.) and the devices used to define the incident X-ray beam. The limitations imposed by the special environment are illustrated in section 3 with the example of a diamond anvil high pressure cell. In section 4 the two diffraction methods - angle dispersive and energy, dispersive - are briefly recalled. In section 5, the flexibility of a S.R. source is discussed in some detail with the example of diffraction studies of samples in a diamond anvil high pressure cell. Similarly the advantage of S.R. for absorption studies with samples at high pressures are briefly mentioned in section 7. A general conclusion is made in section 8 . 


\section{Elements of synchrotron radiation [1].}

Electrons (positrons) travelling with a velocity close to that of light along a bent trajectory emit electromagnetic radiation called synchrotron radiation (S.R.). In practice S.R. is produced by high energy electrons (positrons) stored in a very high vacuum horizontal " torus " and forced onto a closed orbit by dipole magnets (Fig. 1). The " torus " is composed of arcs of a circle, placed in the magnetic field, and straight sections joining the arcs of the circle. These machines are called storage rings.

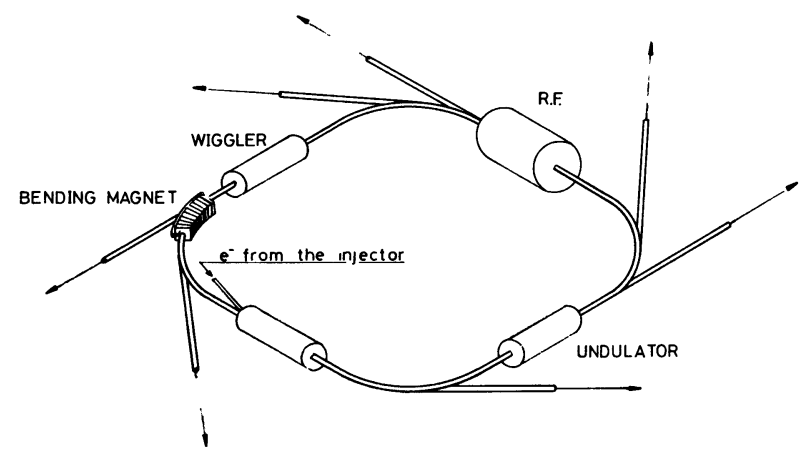

Fig. 1. - Main components of an electron storage ring.

The radiation is emitted tangential to the arcs and is very well collimated in the vertical plane (Fig. 2). The spectral distribution of the radiation is depicted in figure 3, where the spectral flux (called in short flux) is defined as

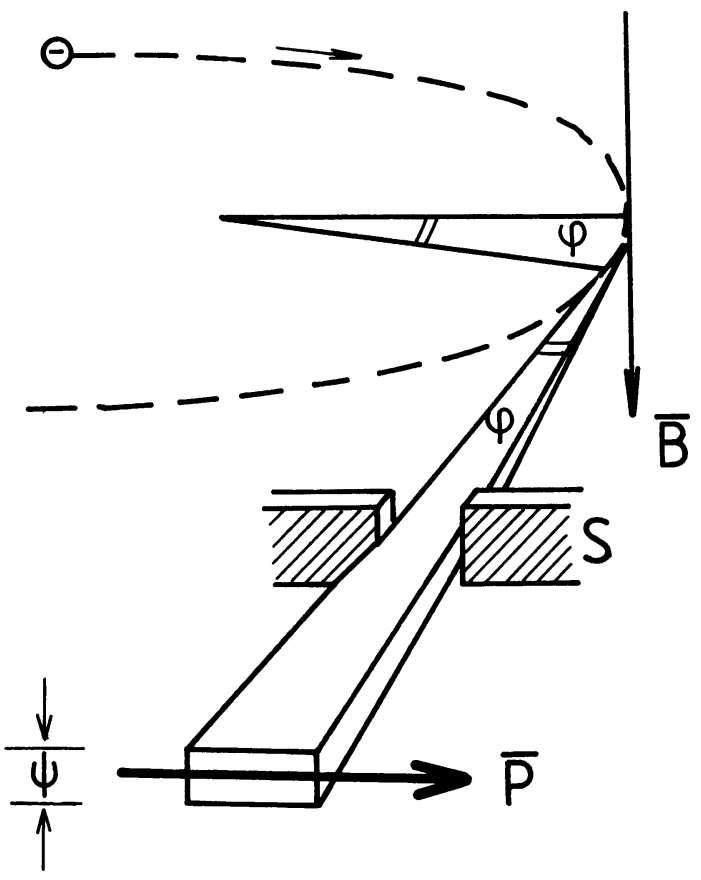

Fig. 2. - Synchrotron radiation emitted by a relativistic electron travelling on a curved trajectory. $\overline{\mathrm{B}}:$ magnetic field perpendicular to the electron orbit plane. $\psi$ : natural opening angle in the vertical plane. $\overline{\mathbf{P}}$ : polarization. The slit $\mathrm{S}$ defines the length of the arc (angle $\varphi$ ) from which the radiation is taken.

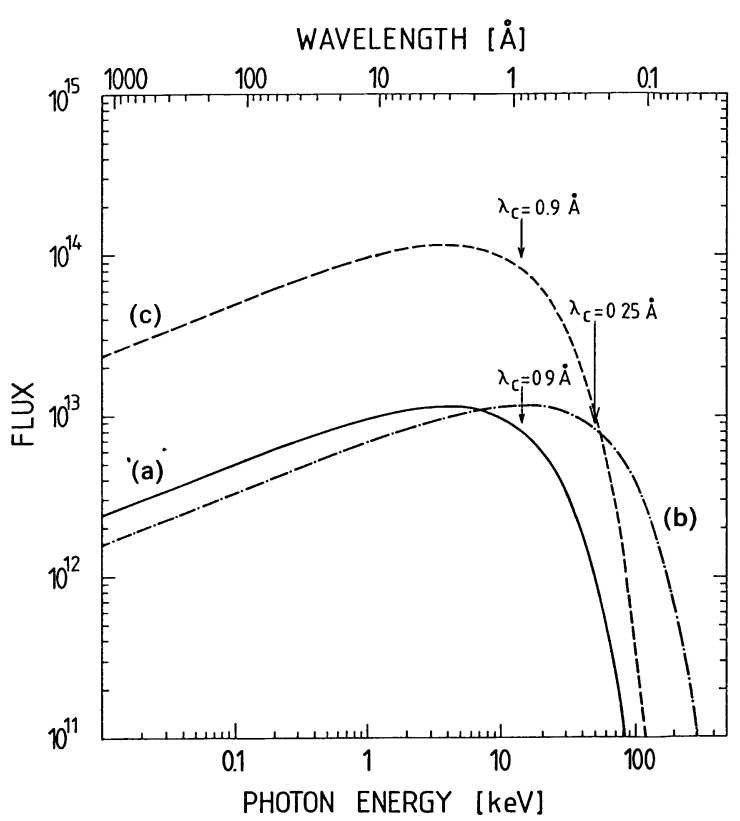

Fig. 3. - Spectral distributions of synchrotron radiation emitted by electrons whose trajectory is determinated by (a) a dipole magnet, (b) a wavelength shifter and (c) a multipole wiggler. (Data for the proposed ESRF.)

number of photons/s

$\overline{\text { mrad horizontal } 0.1 \% \text { relative band width }(\Delta \lambda / \lambda)}$

integrated in the vertical plane. As can be seen from figure 3 the spectral distribution is continuous. It is characterized by the so called characteristic wavelength $\lambda_{\mathrm{c}}$ which divides the spectrum into two parts of equal radiated power. It is related to the parameters of the storage ring through the following equations :

$$
\lambda_{\mathrm{c}}=5.589 \frac{\rho}{E^{3}}=\frac{18.64}{E^{2} B}
$$

where $\lambda_{\mathrm{c}}$ is in $\AA$, the bending radius $\rho$ in meters, the electron energy in $\mathrm{GeV}$ and the magnetic field in tesla. For example, $E=5 \mathrm{GeV}$ and $B=0.75 \mathrm{~T}$ gives $\rho=20 \mathrm{~m}$ and $\left.\lambda_{\mathrm{c}}=0.89 \AA{ }^{1}\right)$.

For an ideal electron orbit, the emitted radiation is very well collimated in the vertical plane and the opening angle $\psi$ is approximately equal to $\gamma^{-1}$, where

$$
\gamma=\frac{E}{\text { rest mass of an electron }} \text {. }
$$

For the example given above $\psi=10^{-4}$ radians $=0.1$ mrad $\sim 20$ seconds of an arc.

In real storage rings, the electron orbit is not ideal and the opening angle $\psi$ might be a bit larger but still very small. The small divergence of the beam

( $\left.{ }^{1}\right)$ These are the parameters of the proposed European Synchrotron Radiation Facility. 
in the vertical plane results in a high spectral brightness (called in short brightness) defined as :

$$
\frac{\text { number of photons } / \mathrm{s}}{(\mathrm{mrad})^{2} 0.1 \% \text { relative band width }(\Delta \lambda / \lambda)} \text {. }
$$

The high brightness of S.R. plays an important role in experiments with small samples, e.g. in high pressure cells, in particular for " white " beam experiments. It is several orders of magnitude larger than that of Bremsstrahlung produced by conventional X-ray tubes.

For experiments in which a monochromatic beam is focused at the sample (by means of mirrors and/or bent single crystals) as, for example, in the case of a sample in a diamond anvil cell, the true figure of merit of the S.R. source is the brilliance defined as

number of photons/s

$\overline{(\mathrm{mrad})^{2}(\mathrm{~mm})^{2} 0.1 \% \text { relative band width }(\Delta \lambda / \lambda)}$.

As follows from equation (1), one can decrease $\lambda_{\mathrm{c}}$ by increasing the magnetic field $B$, and thus shift the whole spectrum toward shorter wavelengths. This can be done locally in a straight section of the " torus ". Such a device is called a single bump wiggler or a wavelength shifter. An example of a spectrum of radiation emitted from a wavelength shifter is shown in figure 3.

At present also periodic multipole magnets are being developed forcing the electrons to make wiggles as shown in figure 4 . These magnets are placed in straight sections of a storage ring and they are called multipole

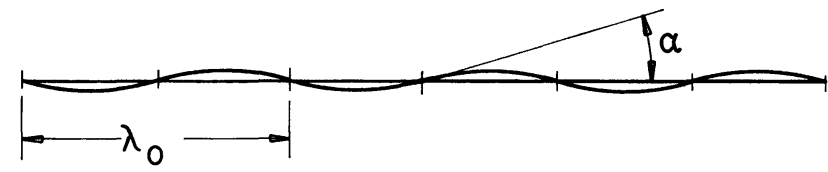

Fig. 4. - Trajectory of electrons within a multipole wiggler or undulator.

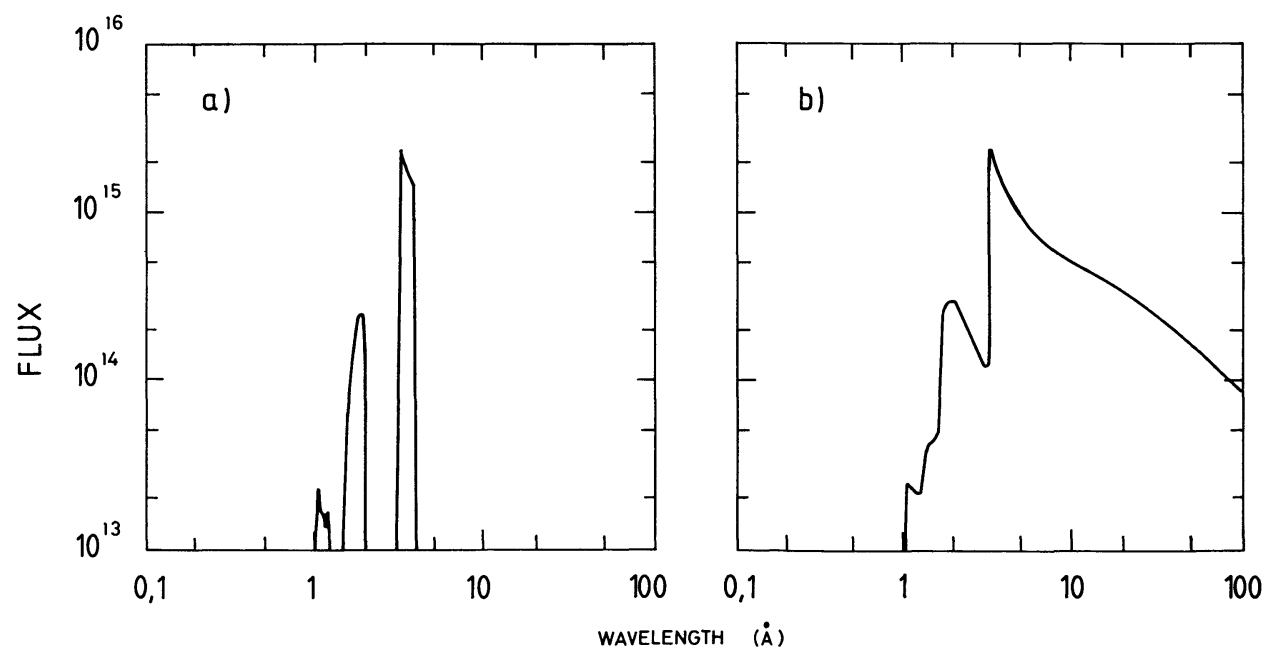

Fig. 5. - Spectrum produced by an undulator with $K=0.5$; (a) observed through a small pinhole on axis, (b) angle integrated, i.e. without the pinhole. wigglers or undulators depending on their characteristic features. They are best characterized by the so called deflection (or magnetic field) parameter

$$
K=\alpha \cdot \gamma=0.0934 B_{0} \lambda_{0}
$$

where $B_{0}$ is the peak magnetic field in tesla, $\lambda_{0}$ is the magnet spatial period in millimeters and $\alpha$ is the maximum deflection angle.

A sharp distinction does not exist between multipole wigglers and undulators. For $K \gg 1$ the device is called a multipole wiggler. With a magnetic field the same as in the bending magnets it does not shift the spectrum but increases the flux by a factor equal roughly to the number of poles (Fig. 3). However, by changing the magnetic field of the multipole wiggler the spectrum can also be shifted.

For $K$ less than 1 or of the order of 1 strong interference effects occur which result in a spectrum of the emitted radiation consisting of one (for $K \ll 1$ ) or several (for $K \simeq 1$ ) quasi monochromatic lines, called harmonics.

The wavelength of the $i$ th harmonic, $\lambda_{i}$, at observation angle $\theta$ (radians) relative to the axis is given by

$$
\lambda_{i}=\frac{\lambda_{0}}{i 2 \gamma^{2}}\left[1 .+\frac{K^{2}}{2}+\gamma^{2} \theta^{2}\right]
$$

On the axis $(\theta=0)$ we have

$$
\lambda_{i}=\frac{\lambda_{0}}{i 2 \gamma^{2}}\left[1+\frac{K^{2}}{2}\right]
$$

and only odd harmonics are present. However, a finite pinhole defining the angle $\theta$ or spread in the angle of electrons passing through the undulator will result in the presence of even harmonics also. A spectrum for a small finite pinhole is presented in figure 5a for $\gamma=10000$ and $K=0.5$. Figure $5 \mathrm{~b}$ 
shows the angle integrated spectrum from the same undulator, i.e. without the pinhole.

The natural relative band width of the emitted radiation for an ideally parallel electron beam is given by

$$
\frac{\Delta \lambda_{i}}{\lambda_{i}}=\frac{1}{i N}
$$

where $N$ is the number of spatial periods.

It follows, however, from equation (4) that due to the dependence of the wavelength on the observation angle the width of the observed line is broadened relative to its natural value given by equation (6). A broadening may occur also due to the divergence of the electron beam. A detailed discussion of these phenomena is beyond the scope of this paper.

The photon brightness emitted by a parallel beam of electrons at the $i$ th harmonic in the forward direction is proportional to $N^{2} \gamma^{2}$ and the electron current. It is much larger than one can obtain from bending magnets or multipole wigglers.

\section{Limitations imposed by the special environment.}

We discuss sample environment problems using the example of synchrotron X-ray diffraction studies of samples in a diamond anvil high pressure cell. Figure 6 shows the principle of such a cell. The sample is placed in a small hole $0.1-0.3 \mathrm{~mm}$ in diameter made in

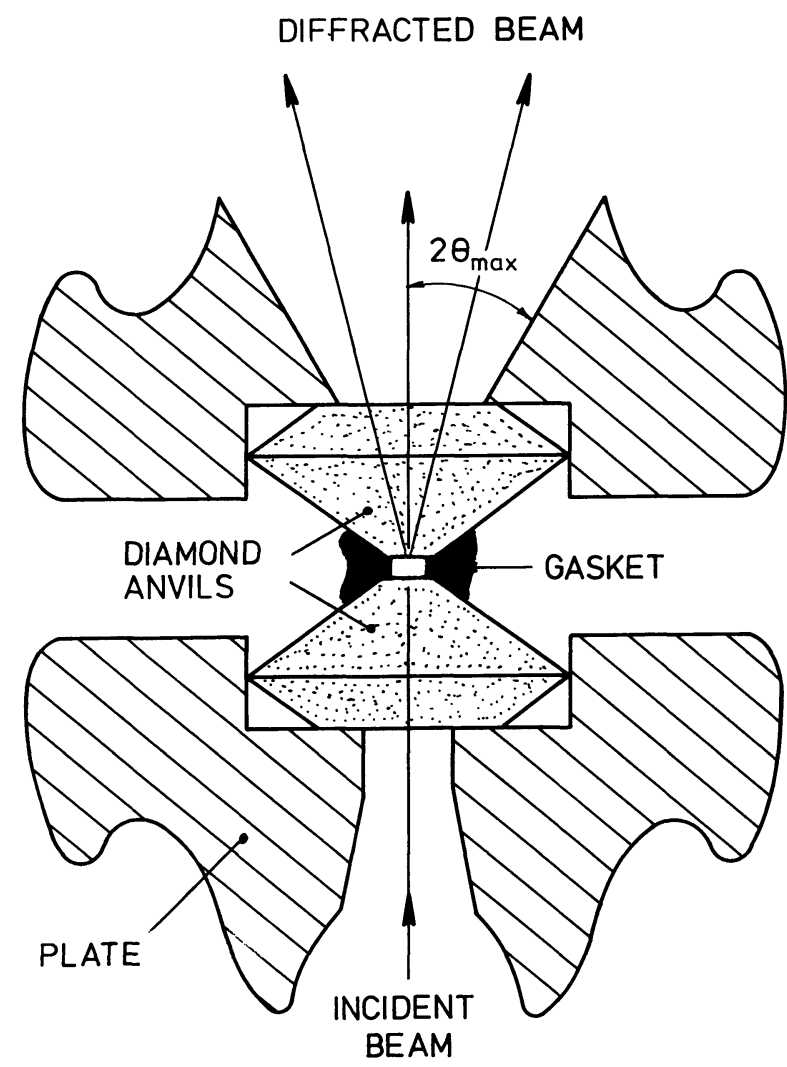

Fig. 6. - Principle diagram of a diamond anvil high pressure cell. an $0.01-0.1 \mathrm{~mm}$ thick metal foil (gasket). The pressure is exerted by the two flat diamond faces pressed against each other.

The main limitations imposed by the high pressure cell on X-ray diffraction studies are the following :

a) the absorption of the incident and diffracted beams by the diamonds,

b) the limitation in the scattering angle,

c) the small cross-section and the small volume of the sample.

As will be shown in sections 5-6, due to the flexibility of a modern synchrotron radiation source, discussed in section 2 , these limitations can be minimized.

\section{Diffraction techniques [2].}

As is well known, there are two diffraction techniques for powdered samples, namely, the angle-dispersive and the energy-dispersive diffraction.

In the angle-dispersive method a monochromatic beam of wavelength $\lambda_{0}$ is selected from the white SR beam by means of a crystal monochromator and the intensity of the beam diffracted by the powdered sample is measured as a function of the scattering angle $2 \theta$. In the energy-dispersive method [2] the scattering angle $2 \theta_{0}$ is fixed and the white SR beam is impinging on the sample. The wavelength (photon energy) composition of the diffracted beam is analysed by means of a solid state detector (SSD). Because of the fixed scattering angle the energy-dispersive method is especially suitable for diffraction studies of samples in special environments and is at present widely used for high pressure research.

The Bragg equations for the two methods are :

$$
d_{H}=\lambda_{0} / 2 \sin \theta_{H}
$$

for angle-dispersive diffraction, and

$$
d_{H}=\lambda_{H} / 2 \sin \theta_{0}=6.199 \mathrm{keV} . \AA / E_{H} \cdot \sin \theta_{0}
$$

for energy-dispersive diffraction, $\left(d_{H}\right.$ denotes the interplanar spacing, $E_{H}$ the photon energy and $H$ the set of reflection indices $H K L$ ).

For the purpose of our discussion, the integrated intensities [3] can be expressed conveniently in the following way :

angle-dispersive diffraction :

$$
P_{H}^{(\mathrm{a})}=C_{\mathrm{a}} \frac{i\left(\lambda_{0}\right) \lambda_{0}^{4} T\left(\lambda_{0}\right)}{\sin \theta_{H} \cdot \sin 2 \theta_{H}} R^{(\mathrm{a})}\left\{j|F|^{2}\right\}_{H}
$$

energy-dispersive diffraction :

$$
P_{H}^{(\mathrm{e})}=C_{\mathrm{e}} \frac{i\left(\lambda_{H}\right) \lambda_{H}^{4} T\left(\lambda_{H}\right)}{\sin \theta_{0} \cdot \sin 2 \theta_{0}} R^{(\mathrm{e})}\left\{j|F|^{2}\right\}_{H}
$$

with :

$$
R^{(\mathrm{a})}=\Delta \lambda_{0} / \lambda_{0} \quad \text { and } \quad R^{(\mathrm{e})}=\cot \theta_{0} \Delta \theta_{0}
$$


where $i(\lambda)$ is the number of photons $/ \mathrm{s} . \mathrm{cm}^{2}$ and per unit interval of wavelength incident on the sample, $T(\lambda)$ the transmission factor, $\Delta \theta_{0}$ the overall divergence of the beam in the energy dispersive method, $C$ a constant depending on the geometry of the experiment, sample volume and unit cell volume, $j$ is the multiplicity factor and $F_{H}$ the structure factor. $R^{(a)}$ and $R^{(\mathrm{e})}$ determine the relative wavelength band widths related to reflections in both methods, respectively. In order to simplify our discussion we assume that $R^{(a)}=R^{(\mathrm{e})}$. For the same reason we neglect the polarization factors and detector efficiencies.

We are, of course, interested only in the $j_{H}\left|F_{H}\right|^{2}$ values, but we measure, as can be seen from equations (8), these values « modulated " by factors

$$
M_{H}^{(\mathrm{a})}=\frac{i\left(\lambda_{0}\right) \lambda_{0}^{4} T\left(\lambda_{0}\right)}{\sin \theta_{H} \cdot \sin 2 \theta_{H}} \quad \text { and } \quad M_{H}^{(\mathrm{e})}=\frac{i\left(\lambda_{H}\right) \lambda_{H}^{4} T\left(\lambda_{H}\right)}{\sin \theta_{0} \cdot \sin 2 \theta_{0}}
$$

in the angle-dispersive and energy-dispersive methods, respectively. In the next section we discuss the dependence of these modulation factors on wavelength and angle.

\section{Flexibility of the S.R. source.}

We discuss first the dependence of $i(\lambda) \lambda^{4} T(\lambda)$ on wavelength, and in order to simplify the discussion further we do not take into account the absorption in the sample.

Figure 7 shows, as an example, $i(\lambda)$ and $i(\lambda) \lambda^{4}$ at

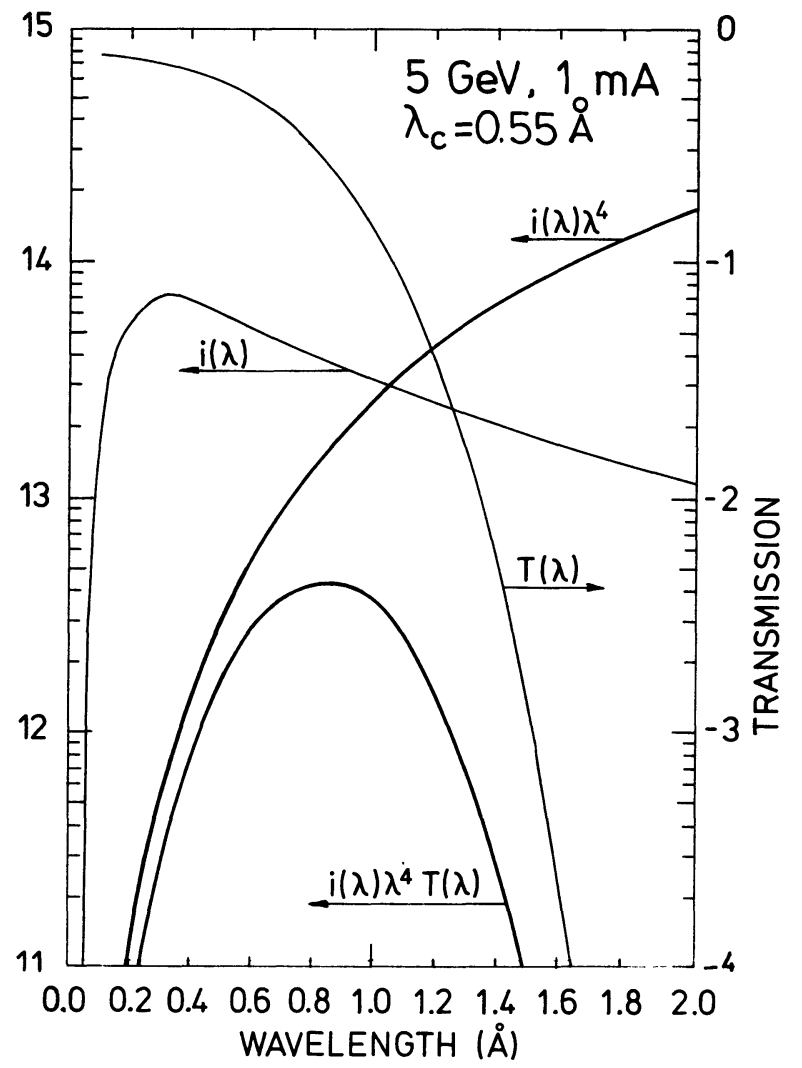

Fig. 7. $-i(\lambda), i(\lambda) \lambda^{4} T(\lambda)$ and $i(\lambda) \lambda^{4} T(\lambda)$ as functions of wavelength of synchrotron radiation with a characteristic wavelength of $0.55 \AA$ (see text). the position of the sample located in the electron orbit plane $40 \mathrm{~m}$ from the bending magnet source point of DORIS ( $5 \mathrm{GeV}, 1 \mathrm{~mA}, R=12.12 \mathrm{~m}, \lambda_{\mathrm{c}}=0.54 \AA$ ).

The figure also shows $T(\lambda)$ for an empty cell composed of two diamonds, each $2.5 \mathrm{~mm}$ thick. We notice that $i(\lambda) \lambda^{4}$ favours long wavelengths and $T(\lambda)$ the short ones, as expected. As a result $i(\lambda) \lambda^{4} T(\lambda)$ reaches a maximum at about $0.8 \AA$ and falls off very rapidly on both sides.

The character of the $i(\lambda) \lambda^{4} T(\lambda)$ curve does not change if one uses a multipole wiggler source instead of a bending magnet source of the same critical wavelength, although all values of $i(\lambda) \lambda^{4} T(\lambda)$ increase by a constant factor equal roughly to the number of poles, resulting in a decrease in measuring time. A singlebump wavelength-shifter, however, shifts the curve toward shorter wavelength, as can be seen from the example in figure 8. Accepting a decrease at long wavelengths one obtains a gain at short wavelengths. Thus $i(\lambda) \lambda^{4} T(\lambda)$ can be tailored according to the requirements of the experiments.

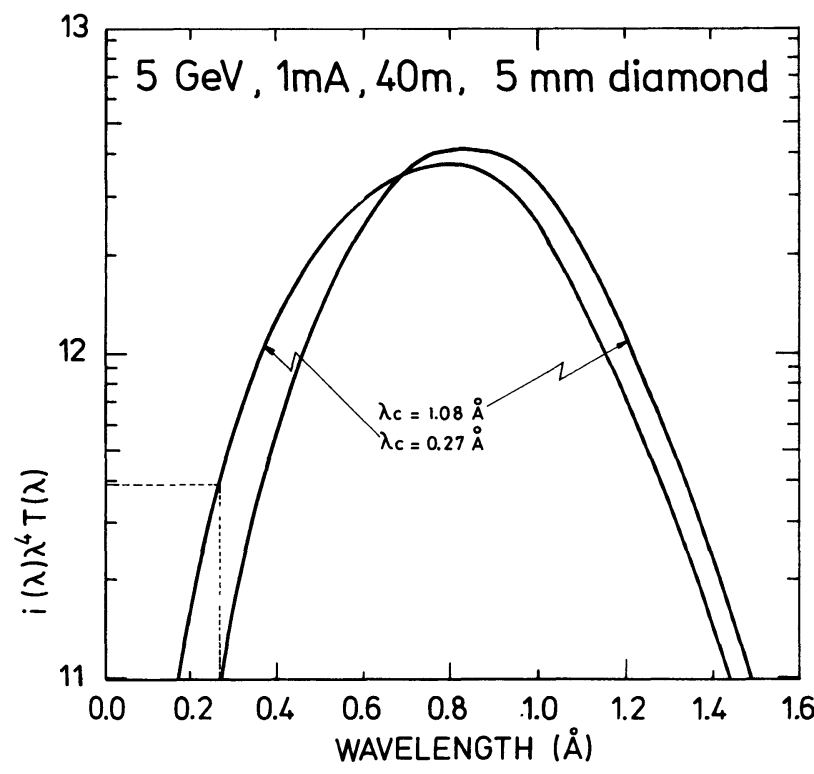

Fig. 8. $-i(\lambda) \lambda^{4} T(\lambda)$ as a function of wavelength for synchrotron radiation of two critical wavelengths, $0.27 \AA$ and $1.08 \AA$.

We discuss now the influence of the modulation factor on the X-ray pattern, beginning with the energydispersive method. Figure 9 shows, as an example, $M^{(\text {e) }}$ as a function of the interplanar spacing $d_{H}$ for two scattering angles, $10^{\circ}$ and $20^{\circ}$. We notice that the modulation factor changes rapidly with the interplanar spacing. Thus the accuracy of measurements due to the statistics is very different for different parts of the pattern.

In the angle-dispersive method one might tend to use a wavelength corresponding to the maximum of the $i(\lambda) \lambda^{4} T(\lambda)$ curve. However, as can be seen from 


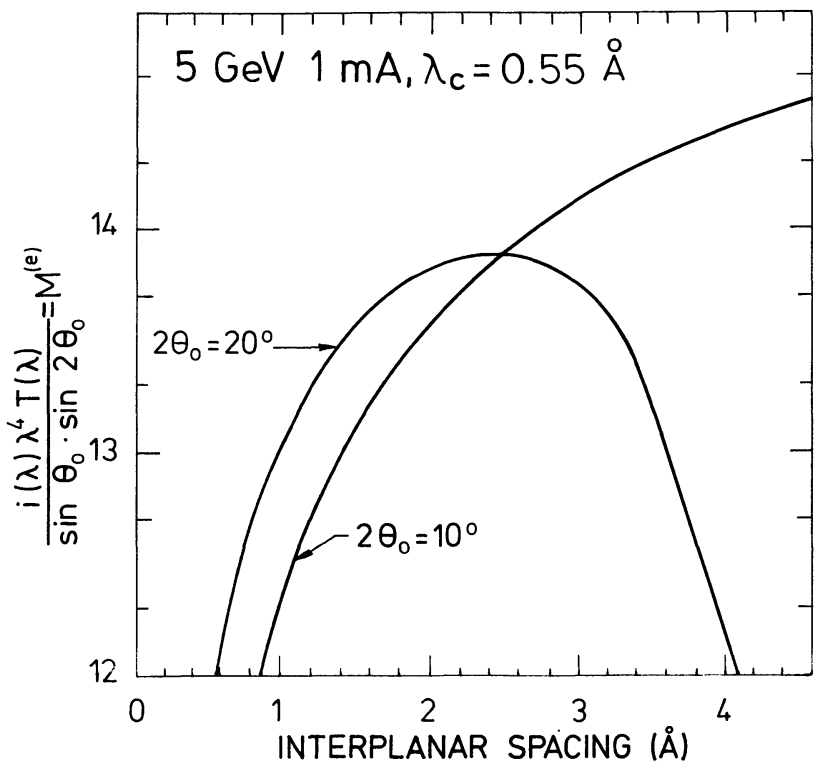

Fig. 9. - The modulation factor $M^{(\mathrm{e})}$ for the energy-dispersive method as a function of the interplanar spacing for two scattering angles, $10^{\circ}$ and $20^{\circ}$.

figure 10 the limitation in the scattering angle puts a lower limit on the range of measured interplanar spacings. If it is not possible to increase $2 \theta_{\max }$ by a proper design of the pressure cell, then one is forced to use a shorter wavelength with a smaller $i\left(\lambda_{0}\right) \lambda^{4} T(\lambda)$ value leading to an increase of the measuring time. As can be seen from figure 10 the modulation factor $M^{(\mathbf{a})}$ changes also rapidly in the angle-dispersive method.

It follows from the above discussion that by shifting the emitted spectrum and increasing the intensity incident on the sample one is able to match the S.R.

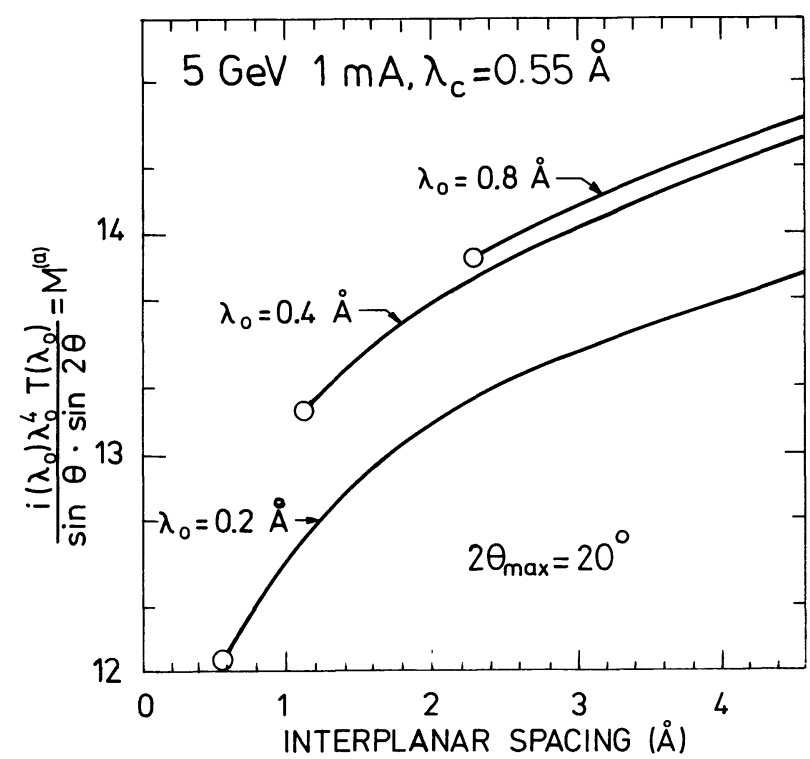

Fig. 10. - The modulation factor $M^{(\mathbf{a})}$ for the angle-dispersive method as a function of the interplanar spacing for three different wavelengths, $\lambda_{0}=0.2 \AA, 0.4 \AA$ and $0.8 \AA$. to the requirements of the experiments in each individual case. For angle-dispersive diffraction one can also use properly designed undulators with their very high brilliance.

\section{The small cross-section and small volume of the sample.}

In order to avoid diffraction lines from the gasket, obscuring the X-ray pattern, and in order to keep the background as low as possible, it is mandatory that the X-ray beam hits the sample only. In recent experiments with pressures up to several hundred of kbar the beam cross-section is typically $0.1 \times 0.1 \mathrm{~mm}^{2}$. In the megabar region these dimensions must be even smaller. This points to both (i) the necessity to use a very good collimated incident beam, and (ii) a high precision of the movements of the high pressure cell in the vertical and horizontal planes. The collimation of the incident beam is determined by (i) the source dimensions, (ii) the sample dimensions, and (iii) the distance between the source and the sample. For dimensions of the source of about $1 \mathrm{~mm}$ in each plane, typical for most of the present machines, a $0.1 \times 0.1 \mathrm{~mm}^{2}$ slit in front of the high pressure cell and a $30 \mathrm{~m}$ source-sample distance, the collimation in the horizontal and vertical plane amounts to about $0.03 \mathrm{mrad}$ or 7 seconds of arc. This

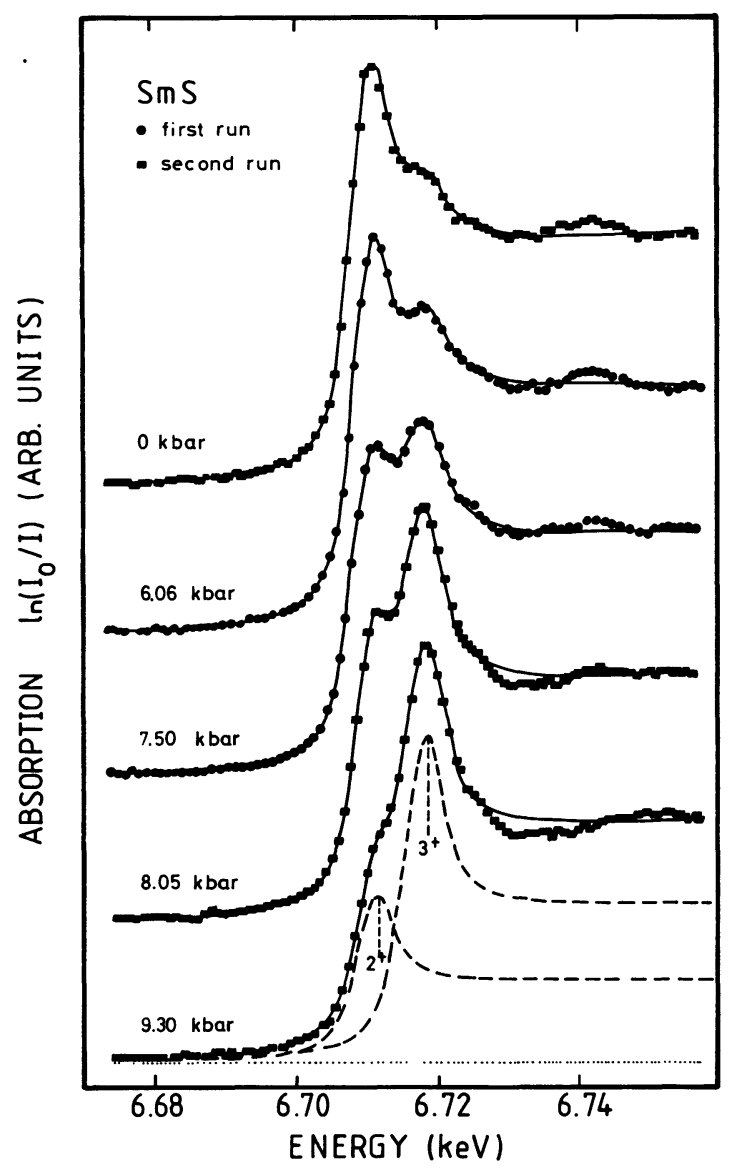

Fig. 11. - Absorption of $\mathrm{SmS}$ at different pressures as function of incident photon energy [3]. 
is satisfactory for most experiments which indicates that - at least at present - high pressure energydispersive diffraction experiments do not need smaller source sizes. It indicates also that as far as collimation is concerned the horizontal and vertical scattering planes are equivalent. They differ, however, with respect to polarization and, as it is well known, a vertical scattering plane is preferable in this respect. For angle-dispersive diffraction one might focus the beam on the sample and then a small source size is important.

The volume of the sample, as already mentioned, is very small $\left(10^{-3}-10^{5} \mathrm{~mm}^{3}\right)$, which especially in powder diffraction results in a very small intensity of the diffracted beams and thus increases the measuring times. This points to both the necessity of using more intense X-ray sources and less time consuming measuring techniques. A synchrotron radiation source and the energy-dispersive method have proved to be a good solution to this problem [2].

It follows from the discussion above that small samples require a source of high brightness for energy-dispersive diffraction and a source of high brilliance for angle-dispersive diffraction.

\section{Absorption studies at high pressures.}

The high brightness of synchrotron radiation sources also allows absorption measurements of samples in special environments. Figure 11 shows, as an example, results of absorption measurements of SmS at different pressures as function of incident photon energy [4]. One notices the shift of the absorption edge with pressure indicating valence change of Samarium from $2^{+}$to $3^{+}$.

\section{Conclusion.}

As could be seen from the above discussion a modern storage ring is an excellent source of $\mathrm{X}$-ray radiation for studies of sampler in special environments. This is due to the fact that the brightness and brilliance of such a S.R. source are much greater than those of Xray tubes and that it can be tailored to the requirements of the experiment.

\section{References}

[1] Handbook on Synchrotron Radiation, edited by E. E. Koch (North-Holland Publishing Company, Amsterdam) 1983. See also European Synchrotron Radiation Facility, Suppl. II : The Machine, eds. D. J. Thompson and N. M. Poole (European Science Foundation, Strasbourg, France) May 1979.

[2] See, for example, Buras, B., Fourme, R. and $\mathbf{K o c h}$, M. H. J., ibid p. 1017-1090 and references therein.
[3] Buras, B., Nucl. Phys. Methods 208 (1983) 563-568.

[4] Frank, K. H., Kainde, G., Feldhaus, J., Wortman, G., Krone, W., MaterliK, G., BACH, H. in Valence Instabilities edited by $\mathrm{P}$. Wrachter and H. Boppart (North-Holland Publishing Company, Amsterdam) 1982, p. 189-194. 